\title{
Quantitative Characterization of Nanometer-Scale Electric Fields via Momentum-Resolved STEM
}

\author{
Andreas Beyer,* Manveer Singh Munde, Saleh Firoozabadi, Damien Heimes, Tim Grieb, \\ Andreas Rosenauer, Knut Müller-Caspary, and Kerstin Volz
}

Cite This: Nano Lett. 2021, 21, 2018-2025

Read Online

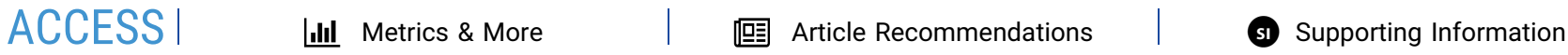

ABSTRACT: Most of today's electronic devices, like solar cells and batteries, are based on nanometer-scale built-in electric fields. Accordingly, characterization of fields at such small scales has become an important task in the optimization of these devices. In this study, with GaAs-based $\mathrm{p}-\mathrm{n}$ junctions as the example, key characteristics such as doping concentrations, polarity, and the depletion width are derived quantitatively using four-dimensional scanning transmission electron microscopy (4DSTEM). The built-in electric fields are determined by the shift they introduce to the center-of-mass of electron diffraction patterns at subnanometer spatial resolution. The method is applied successfully to characterize two $\mathrm{p}-\mathrm{n}$ junctions with different doping concentrations. This

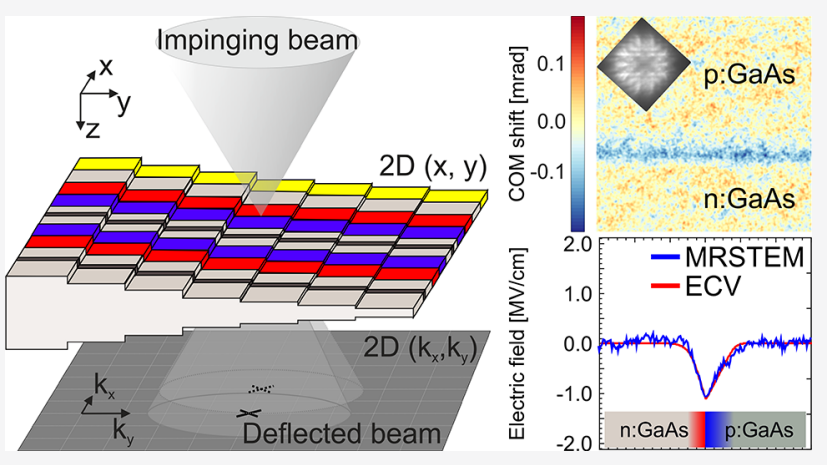
highlights the potential of this method to directly visualize intentional or unintentional nanoscale electric fields in real-life devices, e.g., batteries, transistors, and solar cells.

KEYWORDS: $p-n$ junctions, electric field measurements, transmission electron microscopy, 4DSTEM, momentum-resolved STEM

$\mathrm{T}_{\mathrm{tan}}$ he functionality of most of today's devices, like transistors, batteries, and solar cells, relies on built-in electric fields with strengths in the order of $\mathrm{MV} / \mathrm{cm}$. Their extensions can range from several tens of nanometers down to the atomic scale. The precise control of intentional as well as the detection of any unintentional, parasitic electric fields and their fluctuation is of major importance for a device's functionality. Accordingly, several experimental approaches have been proposed for imaging built-in electric fields such as (scanning) Kelvin probe force microscopy (SKPFM) ${ }^{1,2}$ and electron beam induced current (EBIC) in a scanning electron microscope. ${ }^{3,4}$ The spatial resolution of these methods is limited by the size of the tip used or the diffusion length of the electrons, respectively. Because of its even higher spatial resolution, (scanning) transmission electron microscopy ((S)TEM) has proven a valuable tool for the detection of such nanoscaled electric or also magnetic fields as well. One possible method is, e.g., the TEM-based "Focault" or "displaced aperture" method, which was first reported in 1969 by Titchmarsh et al..$^{5}$ to image Si $\mathrm{p}-\mathrm{n}$ junctions. In this method, the central diffraction spot is observed to distort when the electron beam interacts with the electric field at a $p-n$ junction. Therefore, by carefully positioning an aperture over the distorted spot, an image sensitive to the electric field can be obtained. Shortly after, Merli et al. ${ }^{6}$ reported the imaging of Si $\mathrm{p}-\mathrm{n}$ junctions using the "Fresnel" or "out-of-focus" method. As suggested by its name, this method makes use of a defocused electron beam and can be used in both conventional and scanning modes. ${ }^{7,8}$ However, defocused TEM imaging suffers from interpretability and spatial resolution issues. More recent work has made use of developments in electron holography, not only to image but also to obtain quantitative values for the electrostatic potential at $\mathrm{p}-\mathrm{n}$ junctions in $\mathrm{Si}$ and $\mathrm{GaAs}$ specimens. $^{8-14}$ This method requires a highly specialized setup, in which the electron beam is split to obtain a reference wave that is later interfered with the electron beam after it has interacted with the specimen. Finally, the characterization of a GaAs $p-n$ junction has been carried out using the differential phase contrast (DPC) imaging method. ${ }^{15}$ The application of this technique revealed a high sensitivity to the presence of electric fields and varying dopant concentration, but with relatively low spatial resolution $(\approx 12 \mathrm{~nm})$.

More recently, fast pixelated STEM detectors became available, which allow the acquisition of a full diffraction pattern at each scan point, resulting in four-dimensional STEM (4DSTEM) data sets. ${ }^{16-20}$ These data sets are rich in details and allow to use various techniques, e.g., nano diffraction,

Received: November 16, 2020

Revised: February 17, 2021

Published: February 23, 2021 
ptychography or momentum resolved STEM (MRSTEM) to derive different information. ${ }^{21}$ Using MRSTEM, a present electric field can be measured directly by the momentum transfer, notably by the center-of-mass (COM) shift it induces of the diffraction pattern. The shift angle $\theta$ is directly proportional to the electric field if the electric field does not vary at the scale of the probe: ${ }^{22}$

$$
E=-h \cdot \sin (\theta) / \lambda \cdot v /(t \cdot \mathrm{e})
$$

with $h$ being Planck's constant, $\lambda$ being the wavelength, $v$ the relativistic speed and $e$ the charge of the electron. The $t$ in the equation denotes the distance for which the electron is affected by the field, which corresponds to the thickness of the TEM sample in this case.

So far, this technique has been mainly used to address the electrostatic fields of the atoms within a sample. ${ }^{22-24}$ This requires samples which are as thin as $5 \mathrm{~nm}$ or even twodimensional inherently like $\mathrm{MoS}_{2}$ or $\mathrm{WS}_{2}$. In contrast to this, here we aim for the quantification of longer-range electric fields present in actual devices. Such fields are usually 2 orders of magnitude smaller than the atomic fields, making their detection a real challenge. However, quantitative characterization of these electric fields is of major interest, since they are the basis for the functionality of several devices. To facilitate this task, much higher sample thicknesses of a few hundreds of nanometers are needed to increase the relative contribution of the longer-range fields and to reduce the contributions of the atomic fields.

One suitable benchmark system to test the capabilities of a method to measure electric fields is the $\mathrm{p}-\mathrm{n}$ junction with its well-defined fields mainly determined by the doping concentrations on each side of the junction. In addition, the junction can be formed within the same material, e.g., GaAs, so no interface between two materials is present, which could influence the COM data. ${ }^{25,26}$ So far, the doping levels derived by TEM methods differ from the ones derived by established non TEM-related methods like electrochemical capacitancevoltage (ECV) or Hall measurements. Mostly, the electric fields are underestimated, which is attributed to the presence of dead layers on the sample surfaces. They are supposed to arise from TEM specimen preparation and/or oxidation at the surfaces and do not contribute to the electric field. ${ }^{15,27,28}$ But also a perfect, nonamorphized surface provides surface states, which could result in parasitic electric fields that could actually oppose the fields which should be measured.

Here we use imaging conditions that are also used to perform high-resolution STEM images. For the acquisition of the resulting convergent beam electron diffraction pattern, a fast pixelated-detector is used. We will show that this setup does not deteriorate the detection of the electric field, but on the contrary, the measurements benefit from the higher spatial resolution compared to other methods applied. In order to investigate the capabilities of the experimental setup, we probe two different $\mathrm{p}-\mathrm{n}$ junctions with different doping concentrations and opposite polarity, i.e., $n-p$ and $p-n$. We apply advanced focused ion beam (FIB) TEM-sample preparation, resulting in samples with defined thickness steps which allows the measurement of the thickness-dependence of the electric field in the TEM sample. Moreover, this facilitates a detailed study on the impact of dead layers.

\section{RESULTS}

In a first example, the two $\mathrm{p}-\mathrm{n}$ junctions are investigated for a fixed TEM-sample thickness of approximately $245 \pm 10 \mathrm{~nm}$, which was determined by electron energy loss spectroscopy $(E E L S)^{29}$ and verified by imaging during the FIB preparation. Figure 1a shows a schematic of the prepared TEM sample.
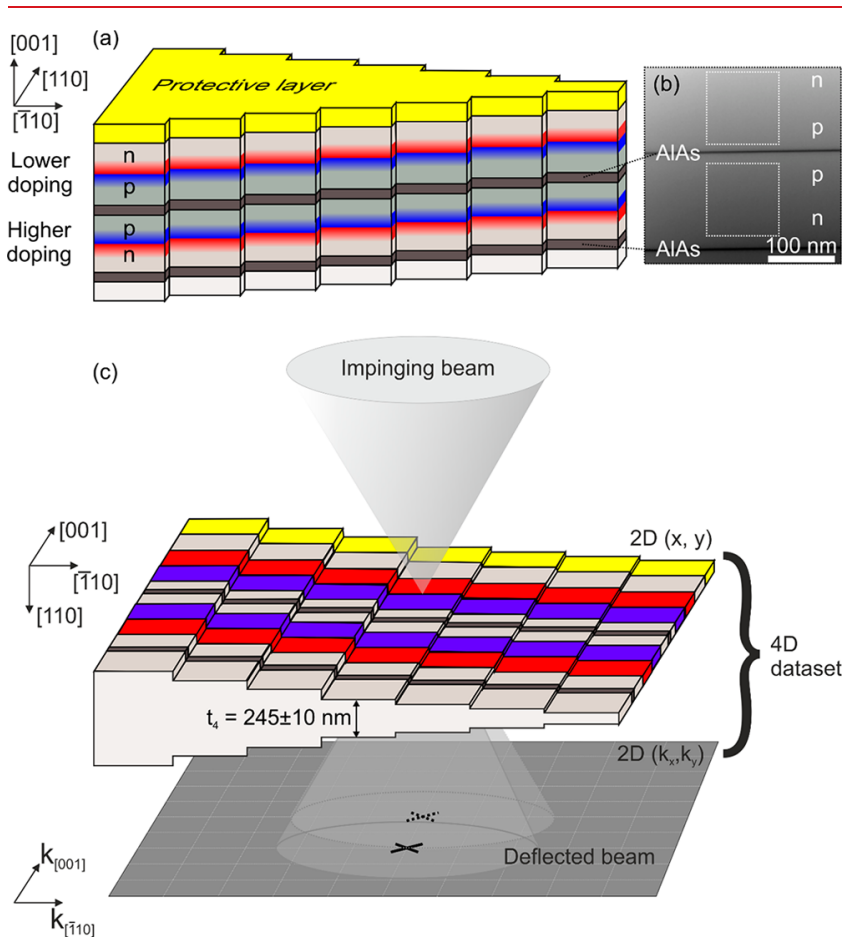

Figure 1. A schematic of the structure of the investigated sample is shown in (a). The optimized FIB preparation allows for the measurement of the two $\mathrm{p}-\mathrm{n}$ junctions at several, here seven, individual sample thicknesses in the TEM. Two AlAs marker layers are visible by their dark contrast in the HAADF overview image of the region of the two $\mathrm{p}-\mathrm{n}$ junctions (b), whereas the actual junctions do not show any contrast. White squares mark the regions where the momentum resolved measurements are carried out. The geometry used in these measurements is illustrated in (c).

Two AlAs marker layers separating the individual junctions from one another are visible by their dark contrast in the high angle annular dark field (HAADF) overview image of the region of the two p-n junctions (b). As expected, the very junctions do not show any contrast in the HAADF image, since the small concentrations of $\mathrm{C}$ and $\mathrm{Te}$ are too little to give any notable change in atomic number detectable by HAADF. The geometry of the experimental MRSTEM setup is illustrated in Figure 1c.

Panels a and $d$ of Figure 2 show higher magnification HAADF images of the higher and lower doped junction, respectively, which were acquired at the positions of the white squares marked in Figure 1b. We want to stress once more that the $\mathrm{p}-\mathrm{n}$ junctions do not exhibit any contrast in the HAADF images. For illustration purposes, the calculated positionaveraged convergent beam electron diffraction pattern (PACBED), i.e., the average of all 65,536 diffraction patterns acquired across the field of view, of each data set is shown as an inset in (a) and (d), respectively. The patterns were rotated by $235^{\circ}$ to align the real space and diffraction space coordinate systems. This rotation was taken into account for the evaluation of the COM data in the following. Figure $2 b$ 

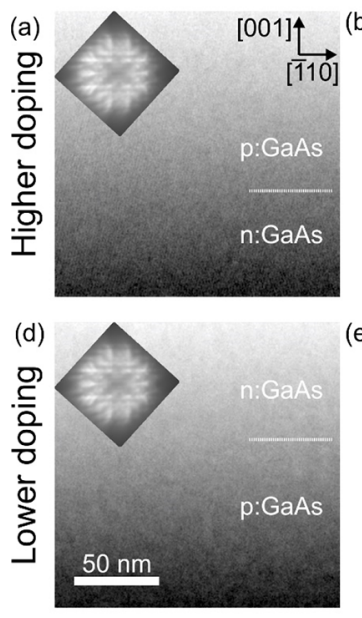

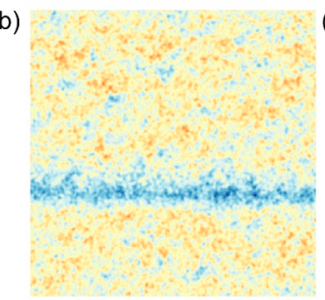

(e)

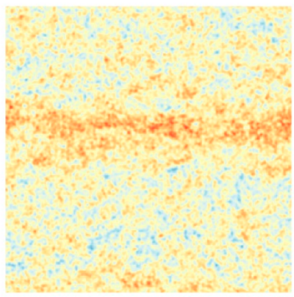

(c)

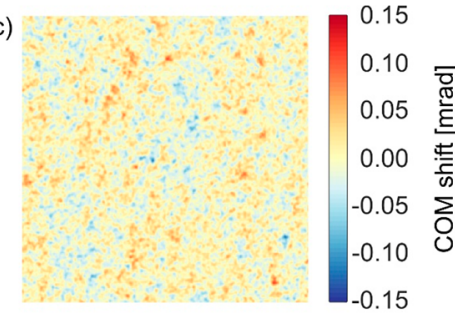

(f)

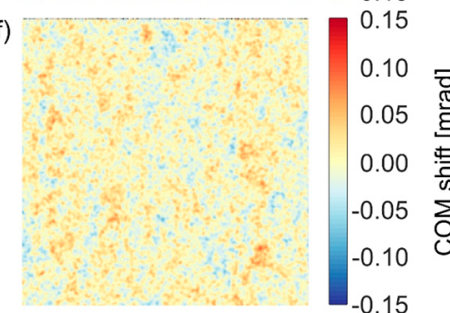

Figure 2. Experimental STEM measurements of higher (top panel) and lower doped (bottom panel) GaAs p-n junctions. The HAADF images of the regions of the higher (a) and lower (d) doped $\mathrm{p}-\mathrm{n}$ junctions do not exhibit contrast at the position of the junction. The intensity gradient from top to bottom is attributed to a slight thickness gradient of the TEM sample, which is in the range of the accuracy of the thickness measurement, i.e., $\pm 10 \mathrm{~nm}$. The corresponding PACBED patterns are shown as insets in (a) and (d), respectively. The patterns were rotated by $235^{\circ}$ to take into account the rotation between real and reciprocal space. The color-coded center of mass signal (COM) of the higher doped junction (b) shows a significant shift opposite of the [001] growth direction at the depletion region. An opposite shift is observed in the signal of the lower doped junction (e) due to the opposite polarity of the junction, i.e., $\mathrm{p}-\mathrm{n}$ instead of $\mathrm{n}-\mathrm{p}$, in the growth direction. No notable shift is observed in the perpendicular direction, which is shown in (c) and (f).
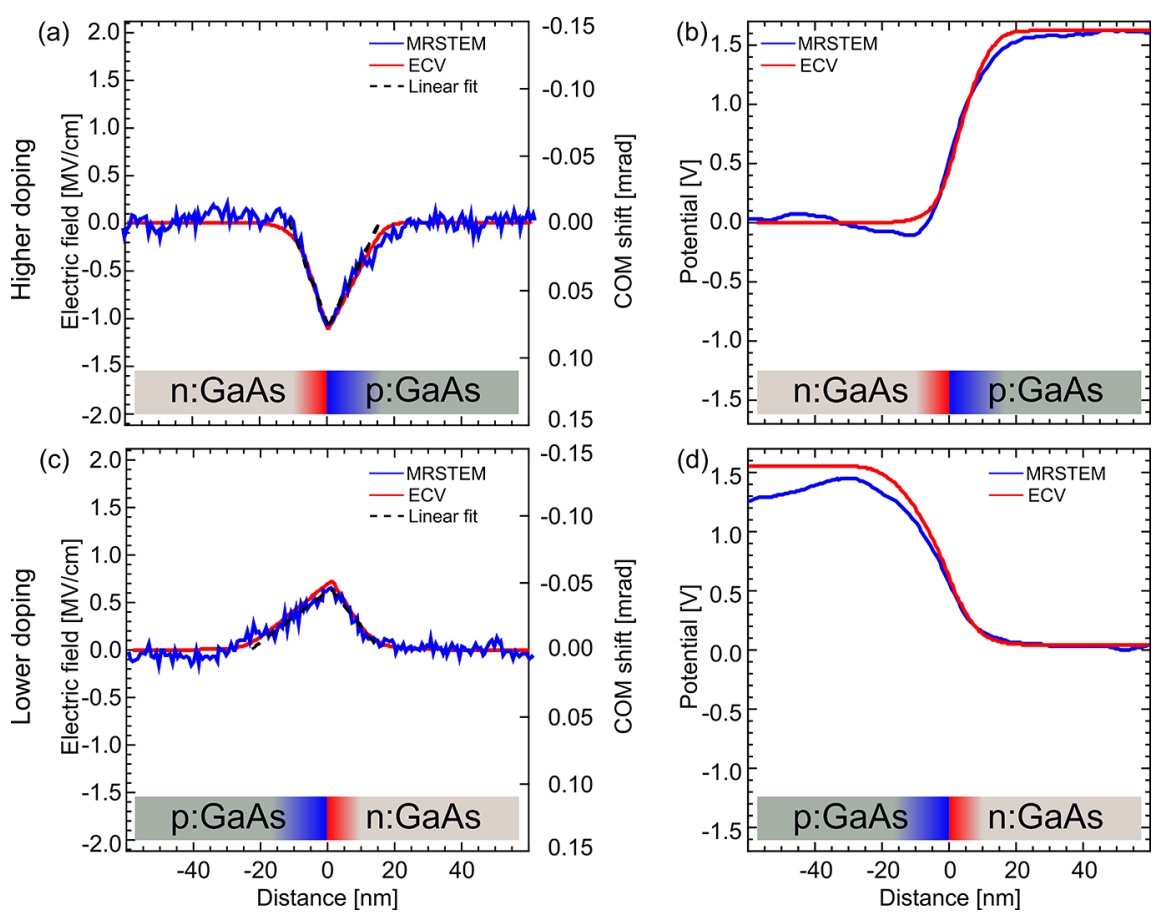

Figure 3. Profile of the COM shift in the growth direction derived from the higher doped $\mathrm{p}-\mathrm{n}$ junction shown as a blue line in (a). The right $y$ axis gives the actual shift measured in mrad, whereas the left $y$ axis gives the corresponding electric field calculated for a sample thickness of $245 \pm 10$ $\mathrm{nm}$. Linear fits to the rise and fall of the peak in electric field are shown as black dashed lines. The electric field/shift calculated from the doping levels determined by ECV is plotted as a red line. The integral over the experimental electric field, which corresponds to the built-in voltage, is shown as a blue line in (b). Again, the curve calculated from the ECV measurements is plotted in red. The corresponding graphs for the lower doped $\mathrm{p}-\mathrm{n}$ junction are collected in $(\mathrm{c})$ and $(\mathrm{d})$.

shows the color coded [001] component of the COM shift at each scan point. The electric fields of the atomic columns lead to a significant low-frequency variation in the COM signal. This is due to the fact that the narrow STEM beam randomly probes different positions of a unit cell of the GaAs crystal, i.e., at an atomic column or at the vacuum in between the columns. A three-dimensional representation of this data set is shown in
Figure SI1 to better visualize the variation induced by the atomic potentials. This atomic signal can be removed by lowpass filtering or averaging over a suitable area in real space. However, even in the unfiltered data, a significant shift in the opposite of the growth direction is visible at the position of the $\mathrm{p}-\mathrm{n}$ junction, which is in line with the expected electric field. The corresponding COM signal for the perpendicular [i 10$]$ 
direction (Figure 2c) does not show any observable shift at the position of the junction, but still the low-frequency variation.

The lower panel of Figure 2 shows the corresponding data for the lower doped $\mathrm{p}-\mathrm{n}$ junction, which intentionally exhibits the switched polarity compared to the higher doped junction, i.e., $\mathrm{p}-\mathrm{n}$ in growth direction instead of $\mathrm{n}-\mathrm{p}$ like before. Accordingly, the opposite deflection of the beam is visible in the [001] COM component, which is shown in Figure 2e. Moreover, the magnitude of the shift is reduced and the width of the region with notable deflection, which corresponds to the depletion region, is wider, in accordance with the reduced doping concentration. In analogy to the higher doped junction, the [110] component of the lower doped junction does not show any significant deflection at the junction.

In the following, the characteristic properties of the $p-n$ junctions will be derived from line profiles, which are collected in Figure 3. By averaging the COM signal along the [ $\overline{1} 10]$ direction, the influences of the atomic electric fields are averaged out, whereas the high spatial resolution along the [001] axis is maintained. A quantitative comparison to the data derived from ECV and by modeling will be carried out later, considering all seven thicknesses available in the TEM sample. Figure 3a depicts the vertically averaged COM signal of the higher doped $\mathrm{p}-\mathrm{n}$ junction as a blue line. The $x$ axis is centered at the position of the maximum absolute shift whose magnitude is $-76 \mu \mathrm{rad}$. Using eq 1 and taking into account the sample thickness of $245 \pm 10 \mathrm{~nm}$, this shift would be caused by a maximum electric field of $1.07 \mathrm{MV} / \mathrm{cm}$. The left $y$ axis shows the strength of the electric field. For visualization purposes, the direction of the COM axis is inverted, since shift and electric field exhibit opposite signs, due to the negative charge of the electron. An asymmetry of the peak is clearly visible, with a steeper slope on the left $(n)$ side compared to the right $(p)$ side of the junction. This reflects the different doping levels $N_{\mathrm{D}}$ and $N_{\mathrm{A}}$ of the $\mathrm{n}$ and $\mathrm{p}$ side of the junction, since the slopes of the fall and rise of the electric field are given by e/ $\varepsilon N_{\mathrm{D}}$ and e/ $\varepsilon N_{\mathrm{A}}$, respectively, ${ }^{30}$ with $\varepsilon$ being the permittivity. Linear fits to the data, which are shown as black dashed lines, yield $6.6 \times$ $10^{18}$ and $5.1 \times 10^{18} \mathrm{~cm}^{-3}$ for $N_{\mathrm{D}}$ and $N_{\mathrm{A}}$, respectively. The width of the peak, which corresponds to the depletion region of the junction, is $27 \mathrm{~nm}$, i.e., 12 and $15 \mathrm{~nm}$ on the $\mathrm{n}$ and $\mathrm{p}$ side, respectively. These values were determined by the intersections of the fits with $y=0$. The course of the plot is in very good quantitative agreement with the expected shift, which was simulated using the nextnano software ${ }^{31}$ using the doping concentrations from ECV as input (red line). Integrating the electric field results in the potential, which is depicted in Figure 3b. The curve resulting from the MRSTEM measurement is shown as a blue line, whereas the ECV curve is shown in red. On the basis of the MRSTEM data, the potential exhibits a step of approximately $V_{0}=1.6 \mathrm{~V}$ across the junction, which corresponds to its built-in voltage. However, there are some discrepancies from the expected step-function-like appearance, since some fluctuations are visible especially on the left, i.e., $\mathrm{n}$ side of the junction. However, it cannot be concluded whether these are caused by experimental noise or an actual variation in built-in potential due to inhomogeneous doping concentrations.

The corresponding plots for the second junction are shown in Figure $3 \mathrm{c}$,d. In accordance with the lower doping concentrations, the maximum shift detected is $46 \mu \mathrm{rad}$ only, which corresponds to a maximum electric field of $-0.64 \mathrm{MV} /$ $\mathrm{cm}$ at the given thickness. Due to the inverted polarity, the shift now points toward the growth direction. The asymmetry between $\mathrm{p}$ and $\mathrm{n}$ regions is nicely observable with a less steep slope on the left $(\mathrm{p})$ side than on the right $(\mathrm{n})$ side. The doping concentrations derived are $1.9 \times 10^{18}$ and $3.1 \times 10^{18}$ $\mathrm{cm}^{-3}$ for $\mathrm{p}$ and $\mathrm{n}$ sides, respectively. The total depletion width is $38 \mathrm{~nm}$ and consists of $24 \mathrm{~nm}$ on the p side (left) and $14 \mathrm{~nm}$ on the $\mathrm{n}$ side (right). The derived potential, which is plotted in Figure $3 \mathrm{~d}$, exhibits a step of $V_{0}=-1.35 \mathrm{~V}$. Please note that the zero level of the potential was chosen on the right-hand side of the junction, where the profile derived by MRSTEM exhibits a plateau.

So far, the two $\mathrm{p}-\mathrm{n}$ junctions were investigated for one sample thickness only. To achieve better statistics and allow for a meaningful comparison of the values derived by MRSTEM to the values derived by ECV, analogue evaluations were carried out for all seven different thicknesses available in the TEM sample. The data derived are collected in Figure 4, where the

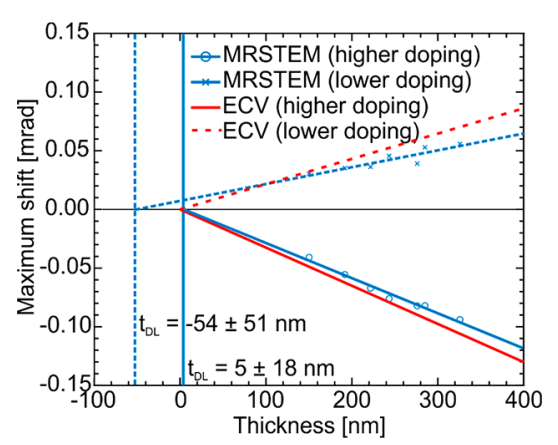

Figure 4. Thickness dependence of the maximum COM shift across the two $\mathrm{p}-\mathrm{n}$ junctions (higher doping: blue circles, lower doping: blue crosses). The blue lines are linear fits to the experimental MRSTEM data. Vertical lines denote the crossing points of these fits with the $x$ axis. The red lines represent the shifts expected from the ECV doping levels.

maximum COM shifts for the higher and lower dopings are shown as blue circles and blue crosses, respectively. Both junctions show a clear linear behavior of the shift with respect to the thickness, with the slope being directly proportional to the electric field, as expected from eq 1. The slope of the higher doped junction is steeper in accordance with the higher doping concentrations. The blue lines represent linear fits to the experimental data, whereas the red lines represent the behavior expected from the doping levels measured by ECV. This thickness-dependent way of displaying the data also allows to address the presence of dead layers on the sample surfaces. If there were any dead layers, the COM shift should be observable only if the sample thickness exceeds their thickness. In other words, the linear fits in Figure 4 should pass $y=0$ at a finite positive thickness, reflecting the dimension of the dead layers. Vertical lines in the respective style mark these crossing points for the higher and lower doped junction, respectively. We find dead layer thicknesses of $t_{\mathrm{DL}}=5 \pm 18 \mathrm{~nm}$ for the higher doped one and an unphysical negative value of $t_{\mathrm{DL}}=-54 \pm 51 \mathrm{~nm}$ for the lower doped one, which is most likely caused by the higher experimental uncertainty at this junction. The errors given are based on the standard deviations of the fit parameters of the linear fits. Considering the error bars, both fits pass through the origin of the coordinate system, which means there are no significant dead layers present on the surfaces. This is a clear improvement over the $112 \mathrm{~nm}$ on each 
Table 1. Comparison of Experimentally Determined and Theoretically Calculated Properties of Both $\mathbf{p}-\mathbf{n}$ Junctions: Maximum Electric Field $E_{\text {Max }}$, Doping Concentrations $N_{\mathrm{A}}$ and $N_{\mathrm{D}}$, Depletion Regions $x_{\mathrm{A}}$ on the $\mathrm{p}$ Side and $x_{\mathrm{D}}$ on the $\mathrm{n}$ Side, Full Width $w$ of the Depletion Region, Built-in Voltage $V_{0}$, and Deviation between in MRSTEM and ECV Values (Delta) in Percent

\begin{tabular}{|c|c|c|c|c|c|c|}
\hline \multirow[b]{2}{*}{ property/method } & \multicolumn{3}{|c|}{ higher doping } & \multicolumn{3}{|c|}{ lower doping } \\
\hline & MRSTEM & ECV & delta $[\%]$ & MRSTEM & ECV & delta [\%] \\
\hline$E_{\mathrm{Max}}[\mathrm{MV} / \mathrm{cm}]$ & $-1.01 \pm 0.04$ & -1.12 & -9.8 & $0.61 \pm 0.07$ & 0.74 & -17.6 \\
\hline$N_{\mathrm{A}}\left[\times 10^{18} \mathrm{~cm}^{-3}\right]$ & $4.5 \pm 0.4$ & $4.8 \pm 0.5$ & -6.3 & $2.0 \pm 0.7$ & $1.8 \pm 0.5$ & 11.1 \\
\hline$N_{\mathrm{D}}\left[\times 10^{18} \mathrm{~cm}^{-3}\right]$ & $6.3 \pm 0.8$ & $7.8 \pm 0.5$ & -21.3 & $2.7 \pm 0.4$ & $4.0 \pm 0.5$ & -32.5 \\
\hline$x_{\mathrm{A}}[\mathrm{nm}]$ & $16 \pm 1$ & 17 & -5.9 & $22 \pm 4$ & 26 & -15.3 \\
\hline$x_{\mathrm{D}}[\mathrm{nm}]$ & $11 \pm 2$ & 10 & 10 & $17 \pm 3$ & 13 & 30.8 \\
\hline$w[\mathrm{~nm}]$ & $28 \pm 2$ & 27 & 3.7 & $39 \pm 3$ & 39 & 0 \\
\hline$V_{0}[\mathrm{~V}]$ & $1.57 \pm 0.12$ & 1.62 & -3.1 & $-1.29 \pm 0.20$ & -1.52 & -15.1 \\
\hline
\end{tabular}

side, which was reported before. ${ }^{15}$ This could be achieved by the improved sample preparation, resulting in negligible amorphous layers. Moreover, surface states, which are also present at a perfect, nonarmorphized surface, also do not play an important role, due to the comparably high TEM-sample thicknesses and doping levels in the junctions.

In total, a good quantitative agreement between MRSTEM and ECV is observable, which will be discussed in more detail in the following.

The average quantities derived from the measurements at different sample thicknesses are collected in Table 1 alongside the values assuming the doping concentrations from ECV measurements.

The experimental variations observable in between the COM measurements for different thicknesses consolidate in the standard deviations given for each property. However, no systematic dependence on thickness was observed. Comparing the experimental and calculated values, it becomes apparent that especially the values for the width of the depletion regions are in excellent agreement. Also, the asymmetries caused by the different doping levels on each side of the respective $p-n$ junction are perfectly retrieved in the experiment. The maximum electric field $E_{\mathrm{Max}}$ of the higher doped junction is -1.7 times higher than the one of the lower-doped junction, which is in good agreement with the factor of -1.5 expected from ECV. However, there is a notable deviation between the experimental and calculated absolute values of $E_{\mathrm{Max}}$. The experimental value and the connected properties $N_{\mathrm{A}}, N_{\mathrm{D}}$, and $V_{0}$ are approximately $10 \%$ lower than expected for the higher doping and 15\% lower than expected for the lower doping. For both junctions, the highest deviation can be observed on the ndoped side. In both cases, the doping level $N_{\mathrm{D}}$ determined by MRSTEM is significantly lower than expected from ECV. In agreement with this, the corresponding depletion width $x_{\mathrm{D}}$ is significantly wider.

However, the differences observed between the values derived by MRSTEM and ECV are in the range of the error bars of each method. A simple broadening of the COM signal within the TEM sample is rather unlikely, since this would also result in a significant widening of the depletion region, which is not observed. Moreover, in this case $V_{0}$ should not be affected at all. Another possible explanation for the observed underestimation of the electric fields could be the presence of dead layers on the TEM sample's surfaces, which do not contribute to the electric field. ${ }^{15,27,28}$ However, we concluded there is no significant impact of dead layers on the surfaces of the measured sample. This is attributed to the optimized sample preparation involving low energy ion milling as well as the fact that the charge carriers are mostly confined to the $p-n$ junctions instead of the sample surfaces due to the high doping.

\section{CONCLUSION}

We have shown that 4DSTEM, and in particular MRSTEM, can be used to characterize electric fields at $p-n$ junctions. The key characteristics, such as doping concentrations, polarity, and width of the depletion region can be derived quantitatively from the COM shift of the convergent beam electron diffraction patterns. We find reasonable agreement of the quantities derived by MRSTEM and the established ECV method for two $\mathrm{p}-\mathrm{n}$ junctions with different doping levels. Due to the high spatial resolution of MRSTEM, potential inhomogeneities in the doping profiles could be revealed. The systematic evaluation of the COM shift at different TEMsample thicknesses suggest that no significant dead layers are present at the sample's surfaces.

In the future, the method presented can be extended to address electric fields at interfaces between different materials. In this case, other factors, which affect the COM, e.g., strain within the heterostructure, have to be considered. Moreover, the methods could allow the direct visualization of intentional or unintentional nanoscale electric fields in real-life devices, e.g., batteries, transistors, and solar cells.

\section{SAMPLE GROWTH AND CHARACTERIZATION}

Two GaAs-based $\mathrm{p}-\mathrm{n}$ junctions with different doping concentrations were grown using metal-organic vapor-phase epitaxy (MOVPE) on semi-insulating GaAs substrate, using TEGa and TBAs as precursors for Ga and As, respectively. The $\mathrm{p}$ and $\mathrm{n}$ regions of each junction were doped by $\mathrm{C}$ and $\mathrm{Te}$ using $\mathrm{CBr}$ and DETe as precursor molecules, respectively. The two junctions exhibit opposite polarities, i.e., $n-p$ and $p-n$, in the growth direction. The junctions are separated by a $4 \mathrm{~nm}$ thick undoped AlAs marker layer so that the junctions can be located by the distinct contrast of AlAs against GaAs in the subsequent TEM measurements. The marker layer is situated $150 \mathrm{~nm}$, respectively, $250 \mathrm{~nm}$ away from the junctions and therefore does not influence their electric fields, which has been verified by solving the Poisson equation of the actual sample structure using the nextnano software. ${ }^{31} \mathrm{We}$ want to emphasize that the AlAs layer is not necessary for the electric field measurement but just helps in conveniently finding the positions of the junctions. A schematic of the sample structure is shown in Figure 1a. The doping concentrations of the junctions were determined via three complementary methods from test structures grown individually. Secondary ion mass 
spectrometry (SIMS) measurements yield the total amount of impurities incorporated, whereas Hall and ECV address the active ones. The carrier concentrations of the higher doped junction determined by ECV are $N_{\mathrm{A}}=4.8 \times 10^{18} \mathrm{~cm}^{-3}$ (SIMS, $8.1 \times 10^{18}$; Hall, $6.3 \times 10^{18}$ ) and $N_{\mathrm{D}}=7.8 \times 10^{18} \mathrm{~cm}^{-3}$ (SIMS, $2.1 \times 10^{19}$; Hall, $\left.2.1 \times 10^{19}\right)$, whereas the concentration of the lower doped junction is $N_{\mathrm{A}}=1.8 \times 10^{18} \mathrm{~cm}^{-3}$ (SIMS, $2.8 \times$ $10^{18}$; Hall, $1.9 \times 10^{18}$ ) and $N_{\mathrm{D}}=4.0 \times 10^{18} \mathrm{~cm}^{-3}$ (SIMS, $6.1 \times$ $10^{18}$; Hall, $\left.8.9 \times 10^{18}\right)$. As expected, the absolute values of the doping levels derived from the individual techniques differ significantly. However, consistently, the carrier concentration in the higher doped junction is a factor of 2-4 higher than the concentration in the lower doped junction and, in both junctions, the $\mathrm{n}$ side is higher doped than the $\mathrm{p}$ side.

TEM sample preparation was carried out using a JEOL JIB 4601 FIB. The viewing direction was chosen as the crystallographic [110] direction. Seven defined thickness steps were created along the electron transparent lamella. During the milling, the acceleration voltage was gradually reduced from 30 down to $5 \mathrm{kV}$. The final polishing was carried out at $900 \mathrm{~V}$ in a Fischione 1040 NanoMill in order to remove remaining amorphous layers on the sample's surfaces. ${ }^{32,33}$ This procedure is expected to reduce the thickness of the electrically dead layers as well. The resulting sample geometry is illustrated in Figure 1a.

The MRSTEM characterization was carried out in a double aberration-corrected JEOL JEM 2200FS operating at $200 \mathrm{kV}$ using a semiconvergence angle of $21 \mathrm{mrad}$, i.e., typical conditions for high resolution STEM (see, e.g., ref 34). This means the diffraction pattern acquired by the pixelated detector is a complex diffraction pattern containing several overlapping disks instead of just the direct beam. Accordingly, the camera length is adjusted in a way that the full area of the camera is covered by the direct beam which results in a comparably low sampling of the reciprocal space. The 4D data sets were acquired using the $\mathrm{pnCCD}^{20}$ in full frame mode with a readout speed of $1000 \mathrm{fps}$.

Conventional HAADF images were acquired simultaneously with the 4D data sets using the conventional ring detector of the TEM. Afterward, complementary EELS measurements were carried out for each of the seven thickness steps, which give $\log$ ratios of $1.66,2.12,2.45,2.69,3.05,3.15$, and 3.60 . This results in thicknesses of 151, 193, 223, 245, 278, 287, and $328 \mathrm{~nm}$, assuming a mean free path of $91 \mathrm{~nm}$ for GaAs, ${ }^{29}$ taking into account that the effective density of scatterers is $\sqrt{ } 2$ higher in the [110] crystallographic direction compared to the $[010]$ one. To further validate these thickness values, we used two independent measurements to determine the thicknesses, i.e., the comparison of the measured HAADF intensity to simulations and the acquisition of SEM images during the FIB preparation. In combination, we estimate the error in thickness to be $\pm 10 \mathrm{~nm}$.

The evaluation of the 4D data sets was carried out using home-written MATLAB routines. The involved postprocessing will be briefly summarized here: first, the COM at each scan point was determined. The actual angle between real and reciprocal space coordinate system, i.e., $235^{\circ}$, was considered. At this angle the [001] COM signal is maximum, the orthogonal [110] COM signal is minimum, and the orientation of the Kikuchi bands visible in the reciprocal space is in agreement with the growth direction determined in real space. The reciprocal units of the diffraction patterns were calibrated using the known width of the GaAs 004 Kikuchi band. A two- dimensional background in the COM data, which is caused by a notable descan (i.e., an unintentional beam tilt while scanning) at the comparably low magnifications, was accounted for.

In this study, MRSTEM is not used to address the electric fields induced by the atomic columns of the sample, like it was shown recently. ${ }^{22-24}$ In contrast to that, the much weaker but longer-ranging built-in fields of actual devices, in this case p-n junctions, shall be measured. Accordingly, the strong impact of the atomic columns has to be removed, which is achieved by averaging the COM signal along the crystallographic [ 110$]$ direction, i.e., the direction perpendicular to the $p-n$ junctions.

\section{ASSOCIATED CONTENT}

\section{SI Supporting Information}

The Supporting Information is available free of charge at https://pubs.acs.org/doi/10.1021/acs.nanolett.0c04544.

Visualization of the impact of atomic electric fields present in the COM data (PDF)

\section{AUTHOR INFORMATION}

\section{Corresponding Author}

Andreas Beyer - Materials Science Centre and Department of Physics, Philipps University Marburg, Marburg 35032,

Germany; (1) orcid.org/0000-0001-6533-0631;

Email: andreas.beyer@physik.uni-marburg.de

\section{Authors}

Manveer Singh Munde - Materials Science Centre and Department of Physics, Philipps University Marburg, Marburg 35032, Germany

Saleh Firoozabadi - Materials Science Centre and Department of Physics, Philipps University Marburg, Marburg 35032, Germany

Damien Heimes - Materials Science Centre and Department of Physics, Philipps University Marburg, Marburg 35032, Germany

Tim Grieb - Institut für Festkörperphysik, Universität Bremen, Bremen 28359, Germany

Andreas Rosenauer - Institut für Festkörperphysik, Universität Bremen, Bremen 28359, Germany

Knut Müller-Caspary - Ernst Ruska-Center for Microscopy and Spectroscopy with Electrons, Forschungszentrum Jülich, Wilhelm-Johnen-Straße, RWTH Aachen University, II. Institute of Physics, Aachen 52074, Germany

Kerstin Volz - Materials Science Centre and Department of Physics, Philipps University Marburg, Marburg 35032, Germany

Complete contact information is available at: https://pubs.acs.org/10.1021/acs.nanolett.0c04544

\section{Author Contributions}

All authors conceived and planned the experiments. M.S.M., S.F., and A.B. carried out the experiments. T.G. carried out the simulations. A.B. took the lead in writing the manuscript. All authors provided critical feedback and helped shape the research, analysis, and manuscript. All authors have given approval to the final version of the manuscript.

\section{Notes}

The authors declare no competing financial interest. 


\section{ACKNOWLEDGMENTS}

A.B., M.S.M., S.F., D.H., and K.V. acknowledge support of the German Research Foundation in the framework of the SFB 1083, from the BMBF in the framework of the "MehrSi project (03SF0525C) as well as of the European Union's Horizon 2020 in the framework of the project microSPIRE (ID: 766955). T.G. and A.R. acknowledge support by the German Research Foundation under contract RO2057/8-3. K.M-C. acknowledges support from the Helmholtz Society (Germany) under contract VH-NG 1317.

\section{REFERENCES}

(1) Buh, G. H.; Chung, H. J.; Kim, C. K.; Yi, J. H.; Yoon, I. T.; Kuk, Y. Imaging of a Silicon Pn Junction under Applied Bias with Scanning Capacitance Microscopy and Kelvin Probe Force Microscopy. Appl. Phys. Lett. 2000, 77 (1), 106-108.

(2) Buh, G. H.; Chung, H. J.; Yi, J. H.; Yoon, I. T.; Kuk, Y. Electrical Characterization of an Operating Si Pn -Junction Diode with Scanning Capacitance Microscopy and Kelvin Probe Force Microscopy. J. Appl. Phys. 2001, 90 (1), 443-448.

(3) Abou-Ras, D.; Schäfer, N.; Baldaz, N.; Brunken, S.; Boit, C. Electron-Beam-Induced Current Measurements with Applied Bias Provide Insight to Locally Resolved Acceptor Concentrations at $\mathrm{p}-\mathrm{n}$ Junctions. AIP Adv. 2015, 5 (7), 077191.

(4) Moore, J. E.; Affouda, C. A.; Maximenko, S. I.; Jenkins, P. Analytical and Numerical Simulation of Electron Beam Induced Current Profiles in P-n Junctions. J. Appl. Phys. 2018, 124 (11), 113102.

(5) Titchmarsh, J. M.; Lapworth, A. J.; Booker, G. R. A New Method for Investigating the Electric Field Regions of P-n Junctions. Phys. Status Solidi B 1969, 34 (2), K83-K86.

(6) Merli, P. G.; Missiroli, G. F.; Pozzi, G. Observation of $p-n$ Junction in Transmission Electron Microscopy by out-of-Focus Technique. Phys. Status Solidi 1973, 16 (2), K89-K91.

(7) Darlington, E. H.; Valdre, U. Imaging of Weak Lorentz Objects ( $\mathrm{p}-\mathrm{n}$ Junctions) by High Voltage Fresnel TEM and STEM. J. Phys. E: Sci. Instrum. 1975, 8 (4), 321-324.

(8) Sasaki, H.; Otomo, S.; Minato, R.; Yamamoto, K.; Hirayama, T. Direct Observation of Dopant Distribution in GaAs Compound Semiconductors Using Phase-Shifting Electron Holography and Lorentz Microscopy. Microscopy 2014, 63 (3), 235-242.

(9) Frabboni, S.; Matteucci, G.; Pozzi, G. Observation of Electrostatic Fields by Electron Holography: The Case of ReverseBiased $\mathrm{p}-\mathrm{n}$ Junctions. Ultramicroscopy 1987, 23 (1), 29-37.

(10) McCartney, M. R.; Smith, D. J.; Hull, R.; Bean, J. C.; Voelkl, E.; Frost, B. Direct Observation of Potential Distribution across $\mathrm{Si} / \mathrm{Si} \mathrm{p}-$ n Junctions Using Off-axis Electron Holography. Appl. Phys. Lett. 1994, 65 (20), 2603-2605.

(11) Twitchett-Harrison, A. C.; Dunin-Borkowski, R. E.; Midgley, P. A. Mapping the Electrical Properties of Semiconductor Junctions-the Electron Holographic Approach. Scanning 2008, 30 (4), 299-309.

(12) Anada, S.; Yamamoto, K.; Sasaki, H.; Shibata, N.; Matsumoto, M.; Hori, Y.; Kinugawa, K.; Imamura, A.; Hirayama, T. Accurate Measurement of Electric Potentials in Biased GaAs Compound Semiconductors by Phase-Shifting Electron Holography. Microscopy 2019, 68 (2), 159-166.

(13) McCartney, M. R; Dunin-Borkowski, R. E.; Smith, D. J. Quantitative Measurement of Nanoscale Electrostatic Potentials and Charges Using Off-Axis Electron Holography: Developments and Opportunities. Ultramicroscopy 2019, 203, 105-118.

(14) Haas, B.; Rouvière, J. L.; Boureau, V.; Berthier, R.; Cooper, D. Direct Comparison of Off-Axis Holography and Differential Phase Contrast for the Mapping of Electric Fields in Semiconductors by Transmission Electron Microscopy. Ultramicroscopy 2019, 198, 58.

(15) Shibata, N.; Findlay, S. D.; Sasaki, H.; Matsumoto, T.; Sawada, H.; Kohno, Y.; Otomo, S.; Minato, R.; Ikuhara, Y. Imaging of Built-in
Electric Field at a $\mathrm{p}-\mathrm{n}$ Junction by Scanning Transmission Electron Microscopy. Sci. Rep. 2015, 5 (1), 10040.

(16) Müller, K.; Ryll, H.; Ordavo, I.; Ihle, S.; Strüder, L.; Volz, K.; Zweck, J.; Soltau, H.; Rosenauer, A. Scanning Transmission Electron Microscopy Strain Measurement from Millisecond Frames of a Direct Electron Charge Coupled Device. Appl. Phys. Lett. 2012, 101 (21), 212110.

(17) Plackett, R.; Horswell, I.; Gimenez, E. N.; Marchal, J.; Omar, D.; Tartoni, N. Merlin: A Fast Versatile Readout System for Medipix3. J. Instrum. 2013, 8 (01), C01038-C01038.

(18) Müller-Caspary, K.; Oelsner, A.; Potapov, P. Two-Dimensional Strain Mapping in Semiconductors by Nano-Beam Electron Diffraction Employing a Delay-Line Detector. Appl. Phys. Lett. 2015, 107 (7), 072110.

(19) Tate, M. W.; Purohit, P.; Chamberlain, D.; Nguyen, K. X.; Hovden, R.; Chang, C. S.; Deb, P.; Turgut, E.; Heron, J. T.; Schlom, D. G.; Ralph, D. C.; Fuchs, G. D.; Shanks, K. S.; Philipp, H. T.; Muller, D. A.; Gruner, S. M. High Dynamic Range Pixel Array Detector for Scanning Transmission Electron Microscopy. Microsc. Microanal. 2016, 22 (01), 237-249.

(20) Ryll, H.; Simson, M.; Hartmann, R.; Holl, P.; Huth, M.; Ihle, S.; Kondo, Y.; Kotula, P.; Liebel, A.; Müller-Caspary, K.; Rosenauer, A.; Sagawa, R.; Schmidt, J.; Soltau, H.; Strüder, L. A PnCCD-Based, Fast Direct Single Electron Imaging Camera for TEM and STEM. J. Instrum. 2016, 11 (04), P04006-P04006.

(21) Ophus, C. Four-Dimensional Scanning Transmission Electron Microscopy (4D-STEM): From Scanning Nanodiffraction to Ptychography and Beyond. Microsc. Microanal. 2019, 25 (May), $563-582$.

(22) Müller, K.; Krause, F. F.; Béché, A.; Schowalter, M.; Galioit, V.; Löffler, S.; Verbeeck, J.; Zweck, J.; Schattschneider, P.; Rosenauer, A. Atomic Electric Fields Revealed by a Quantum Mechanical Approach to Electron Picodiffraction. Nat. Commun. 2014, 5 (1), 5653.

(23) Hachtel, J. A.; Idrobo, J. C.; Chi, M. Sub-Ångstrom Electric Field Measurements on a Universal Detector in a Scanning Transmission Electron Microscope. Adv. Struct. Chem. Imaging 2018, 4 (1), 10.

(24) Fang, S.; Wen, Y.; Allen, C. S.; Ophus, C.; Han, G. G. D.; Kirkland, A. I.; Kaxiras, E.; Warner, J. H. Atomic Electrostatic Maps of 1D Channels in 2D Semiconductors Using 4D Scanning Transmission Electron Microscopy. Nat. Commun. 2019, 10 (1), 1127.

(25) Müller-Caspary, K.; Grieb, T.; Müßener, J.; Gauquelin, N.; Hille, P.; Schörmann, J.; Verbeeck, J.; Van Aert, S.; Eickhoff, M.; Rosenauer, A. Electrical Polarization in AlN/GaN Nanodisks Measured by Momentum-Resolved 4D Scanning Transmission Electron Microscopy. Phys. Rev. Lett. 2019, 122 (10), 106102.

(26) Beyer, A.; Volz, K. Advanced Electron Microscopy for III/V on Silicon Integration. Adv. Mater. Interfaces 2019, 6, 1801951.

(27) Seki, T.; Sánchez-Santolino, G.; Ishikawa, R.; Findlay, S. D.; Ikuhara, Y.; Shibata, N. Quantitative Electric Field Mapping in Thin Specimens Using a Segmented Detector: Revisiting the Transfer Function for Differential Phase Contrast. Ultramicroscopy 2017, 182, 258-263.

(28) Toyama, S.; Seki, T.; Anada, S.; Sasaki, H.; Yamamoto, K.; Ikuhara, Y.; Shibata, N. Quantitative Electric Field Mapping of a p-n Junction by DPC STEM. Ultramicroscopy 2020, 216, 113033.

(29) Iakoubovskii, K.; Mitsuishi, K.; Nakayama, Y.; Furuya, K. Thickness Measurements with Electron Energy Loss Spectroscopy. Microsc. Res. Tech. 2008, 71 (8), 626-631.

(30) Sze, S. M.; Ng, K. K. Physics of Semiconductor Devices, 3rd ed.; John Wiley \& Sons: Hoboken, 2006.

(31) Birner, S.; Zibold, T.; Andlauer, T.; Kubis, T.; Sabathil, M.; Trellakis, A.; Vogl, P. Nextnano: General Purpose 3-D Simulations. IEEE Trans. Electron Devices 2007, 54 (9), 2137-2142.

(32) Lotnyk, A.; Poppitz, D.; Ross, U.; Gerlach, J. W.; Frost, F.; Bernütz, S.; Thelander, E.; Rauschenbach, B. Focused High- and LowEnergy Ion Milling for TEM Specimen Preparation. Microelectron. Reliab. 2015, 55 (9-10), 2119-2125. 
(33) Cerchiara, R. R.; Fischione, P. E.; Liu, J.; Matesa, J. M.; Robins, A. C.; Fraser, H. L.; Genc, A. Raising the Standard of Specimen Preparation for Aberration-Corrected TEM and STEM. Microsc. Today 2011, 19 (01), 16-19.

(34) Duschek, L.; Kükelhan, P.; Beyer, A.; Firoozabadi, S.; Oelerich, J. O.; Fuchs, C.; Stolz, W.; Ballabio, A.; Isella, G.; Volz, K. Composition Determination of Semiconductor Alloys towards Atomic Accuracy by HAADF-STEM. Ultramicroscopy 2019, $200,84$. 